\title{
Dynamics of quasi-one-dimensional bright and vortex solitons of a dipolar Bose-Einstein condensate with repulsive atomic interaction
}

\author{
Luis E. Young-S. ${ }^{1}$, P. Muruganandam ${ }^{1,2}$, and S. K. Adhikari ${ }^{1} \S$ \\ ${ }^{1}$ Instituto de Física Teórica, UNESP - Universidade Estadual Paulista, 01.140-070 \\ São Paulo, São Paulo, Brazil \\ ${ }^{2}$ School of Physics, Bharathidasan University, Palkalaiperur Campus, Tiruchirappalli \\ 620024, Tamilnadu, India
}

\begin{abstract}
By numerical and variational analysis of the three-dimensional GrossPitaevskii equation we study the formation and dynamics of bright and vortex-bright solitons in a cigar-shaped dipolar Bose-Einstein condensate for large repulsive atomic interactions. Phase diagram showing the region of stability of the solitons is obtained. We also study the dynamics of breathing oscillation of the solitons as well as the collision dynamics of two solitons at large velocities. Two solitons placed side-by-side at rest coalesce to form a stable bound soliton molecule due to dipolar attraction.

PACS numbers: 03.75.Lm,05.45.Yv,05.30.Jp
\end{abstract}

$\ddagger$ anand@cnld.bdu.ac.in

$\S$ Email: adhikari@ift.unesp.br; URL: http://www.ift.unesp.br/users/adhikari/ 
A bright soliton is a self-reinforcing solitary wave that maintains its shape, while traveling at constant speed, due to a cancellation of nonlinear attraction and dispersive effects. Experimentally, bright matter-wave solitons and soliton trains were created in a Bose-Einstein condensate (BEC) of ${ }^{7} \mathrm{Li}$ [1, 2] and ${ }^{85} \mathrm{Rb}$ atoms [3] by turning the atomic interaction attractive from repulsive using a Feshbach resonance (FR) [4] and releasing the BEC in an axially free or an expulsive trap.

Lately, a BEC of ${ }^{52} \mathrm{Cr}$ atoms with a large long-range dipolar interaction has been made [5, 6]. This allows to study the dipolar BEC (DBEC) with variable short-range interaction [5, 6] using a FR [4]. The DBEC has many distinct features [5, 7, 8, 9]. The stability of a DBEC depends not only on the scattering length, but also on the trap geometry [5, 7, 9]. A disk-shaped trap gives a repulsive dipolar interaction and the DBEC is more stable. In contrast, a cigar-shaped trap yields an attractive dipolar interaction and hence may favor a collapse [5, 9, 10]. Collapse instability of solitons in the nonpolynomial Schrödinger equation with dipole-dipole interactions was studied by Gligorić et al. [11]. The controllable short-range interaction with the exotic dipolar interaction makes the DBEC an interesting system for soliton generation in the cigar configuration due to added dipolar attraction and a challenging system for theoretical investigation [12] to study the interplay between the dipolar and short-range interactions.

We suggest cigar-shaped bright and vortex solitons [13] for repulsive short-range interaction in a DBEC free to move along the axial direction and trapped in the radial direction, using the three-dimensional (3D) Gross-Pitaevskii (GP) equation, and study their statics and dynamics. Vortex-bright solitons (called vortex solitons hereafter) are solitons of (quantized) unit angular momentum. In the usual (non-dipolar) BEC, solitons with intrinsic vorticity trapped in the cigar-shaped potential, were considered by Salasnich et al. [14]. Vortex states of higher angular momentum $(l>1)$ are unstable and decay into multiple vortices of angular momentum $\hbar$ [15]. For a conventional BEC without dipole moment, solitons appear only for attractive atomic interaction. Phase plots showing the stability of the solitons for different dipolar and short-range interactions are obtained. We study breathing oscillation and collision of two bright and two vortex solitons as well as the statics and dynamics of the bright solitons using a Gaussian variational approach. Collision of two bright and vortex solitons in nondipolar BEC was studied in [16]. Where applicable, the variational results are found to be in good agreement with numerical results. By reversing the sign of the dipolar interaction, Pedri and Santos [17] studied stable two-dimensional (2D) bright solitons in a disk-shaped DBEC free to move in the radial plane and trapped in the axial direction. These solitons were later generalized to 2D vortex solitons [18]. There has also been study of anisotropic 2D solitons not requiring the sign-reversal of the dipolar interaction [19]. However, special conditions are needed to prepare these 2D solitons, whereas the present one-dimensional (1D) solitons can be formed under very general conditions and hence, are of greater experimental interest. Solitons in the Tonks-Girardeau gas with dipolar interaction was studied by Baizakov et al. [20] in a 1D model. 
Cuevas et al. [21] studied solitons in cigar-shaped DBEC using a reduced quasi-1D toy model [22]. As there is no collapse in 1D (models with cubic nonlinearity as in this case), such study predicts soliton for attractive interaction for an arbitrarily large number of atoms. But non-dipolar BEC bright solitons [23] and vortex solitons [24] in 3D are stable upto a critical number of atoms, beyond which the system collapses. The same is found to be true for a DBEC; viz. the critical numbers reported in figure 1 (a) DBEC soliton.

We study the bright solitons in a DBEC of $N$ atoms, each of mass $m$, using the GP equation: [5]

$$
\begin{aligned}
i \frac{\partial \phi(\mathbf{r}, t)}{\partial t} & =\left[-\frac{1}{2} \nabla^{2}+\frac{\rho^{2}}{2}+4 \pi a N|\phi(\mathbf{r}, t)|^{2}\right. \\
& \left.+N \int U_{d d}\left(\mathbf{r}-\mathbf{r}^{\prime}\right)\left|\phi\left(\mathbf{r}^{\prime}, t\right)\right|^{2} d \mathbf{r}^{\prime}\right] \phi(\mathbf{r}, t),
\end{aligned}
$$

with the dipolar interaction $U_{d d}(\mathbf{R})=3 a_{d d}\left(1-3 \cos ^{2} \theta\right) / R^{3}, \quad \mathbf{R}=\mathbf{r}-\mathbf{r}^{\prime}$ and normalization $\int \phi(\mathbf{r})^{2} d \mathbf{r}=1$. Here, $a$ is the atomic scattering length, $\theta$ the angle between $\mathbf{R}$ and the polarization direction $z$. The length $a_{d d}=\mu_{0} \bar{\mu}^{2} m /\left(12 \pi \hbar^{2}\right)$ measures the strength of dipolar interaction and its experimental value for ${ }^{52} \mathrm{Cr}$ is $15 a_{0}$ [5], with $a_{0}$ the Bohr radius, $\bar{\mu}$ the (magnetic) dipole moment of a single atom, and $\mu_{0}$ the permeability of free space. The trap $\rho^{2} / 2$ acts in the radial $\rho \equiv(x, y)$ direction only. To obtain a quantized vortex of angular momentum $l \hbar, l=1$, around axial $z$ axis, one has to introduce a phase (equal to the azimuthal angle) in the wave function [25. This procedure introduces a centrifugal term $l^{2} / 2 \rho^{2}$ in the GP equation for a vortex and we adopt this method to study vortex solitons. In (1) we use oscillator unit of transverse trap: unit of time is inverse angular frequency $\omega_{\perp}$ taken as $1 \mathrm{~ms}$, unit of length is oscillator length $a_{\perp} \equiv \sqrt{\hbar / m \omega_{\perp}}$ taken as $1 \mu \mathrm{m}$.

Lagrangian density of (1) for bright soliton is [13]

$$
\begin{aligned}
\mathcal{L}= & \frac{i}{2}\left(\phi \phi_{t}^{\star}-\phi^{\star} \phi_{t}\right)+\frac{1}{2}|\nabla \phi|^{2}+\frac{\rho^{2}}{2}|\phi|^{2}+2 \pi a N|\phi|^{4} \\
& +\frac{N}{2}|\phi|^{2} \int U_{d d}\left(\mathbf{r}-\mathbf{r}^{\prime}\right)\left|\phi\left(\mathbf{r}^{\prime}\right)\right|^{2} d \mathbf{r}^{\prime} .
\end{aligned}
$$

For a variational study we use the Gaussian ansatz [26]: $\phi(\mathbf{r}, t)=\exp \left(-\rho^{2} / 2 w_{\rho}^{2}-z^{2} / 2 w_{z}^{2}\right.$ $\left.+i \alpha \rho^{2}+i \beta z^{2}\right) /\left(w_{\rho} \sqrt{w}_{z} \pi^{3 / 4}\right)$ where $w_{\rho}$ and $w_{z}$ are time-dependent widths and $\alpha$ and $\beta$ are time-dependent phases. The effective Lagrangian $L$ (per particle) is

$$
\begin{aligned}
L & \equiv \int \mathcal{L} d \mathbf{r}=\left(w_{\rho}^{2} \dot{\alpha}+\frac{1}{2} w_{z}^{2} \dot{\beta}+2 w_{\rho}^{2} \alpha^{2}+w_{z}^{2} \beta^{2}\right) \\
& +\frac{1}{2}\left(\frac{1}{w_{\rho}^{2}}+\frac{1}{2 w_{z}^{2}}+w_{\rho}^{2}\right)+\mathcal{E}_{\mathrm{dip}},
\end{aligned}
$$

with $\mathcal{E}_{\text {dip }}=N\left[a-a_{d d} f(\kappa)\right] /\left(\sqrt{2 \pi} w_{\rho}^{2} w_{z}\right), f(\kappa)=\left[1+2 \kappa^{2}-3 \kappa^{2} d(\kappa)\right] /\left(1-\kappa^{2}\right), d(\kappa)=$ $\left(\operatorname{atanh} \sqrt{1-\kappa^{2}}\right) / \sqrt{1-\kappa^{2}}, \kappa=w_{\rho} / w_{z}$. In the cigar shape, the dipolar interaction becomes attractive and contributes negatively in $\mathcal{E}_{\text {dip }}$. The Euler-Lagrange equations for parameters $w_{\rho}, w_{z}, \alpha, \beta$ can be used to obtain the following equations for the widths

$$
\ddot{w}_{\rho}+w_{\rho}=\frac{1}{w_{\rho}^{3}}+\frac{1}{\sqrt{2 \pi}} \frac{N}{w_{\rho}^{3} w_{z}}\left[2 a-a_{d d} g(\kappa)\right],
$$



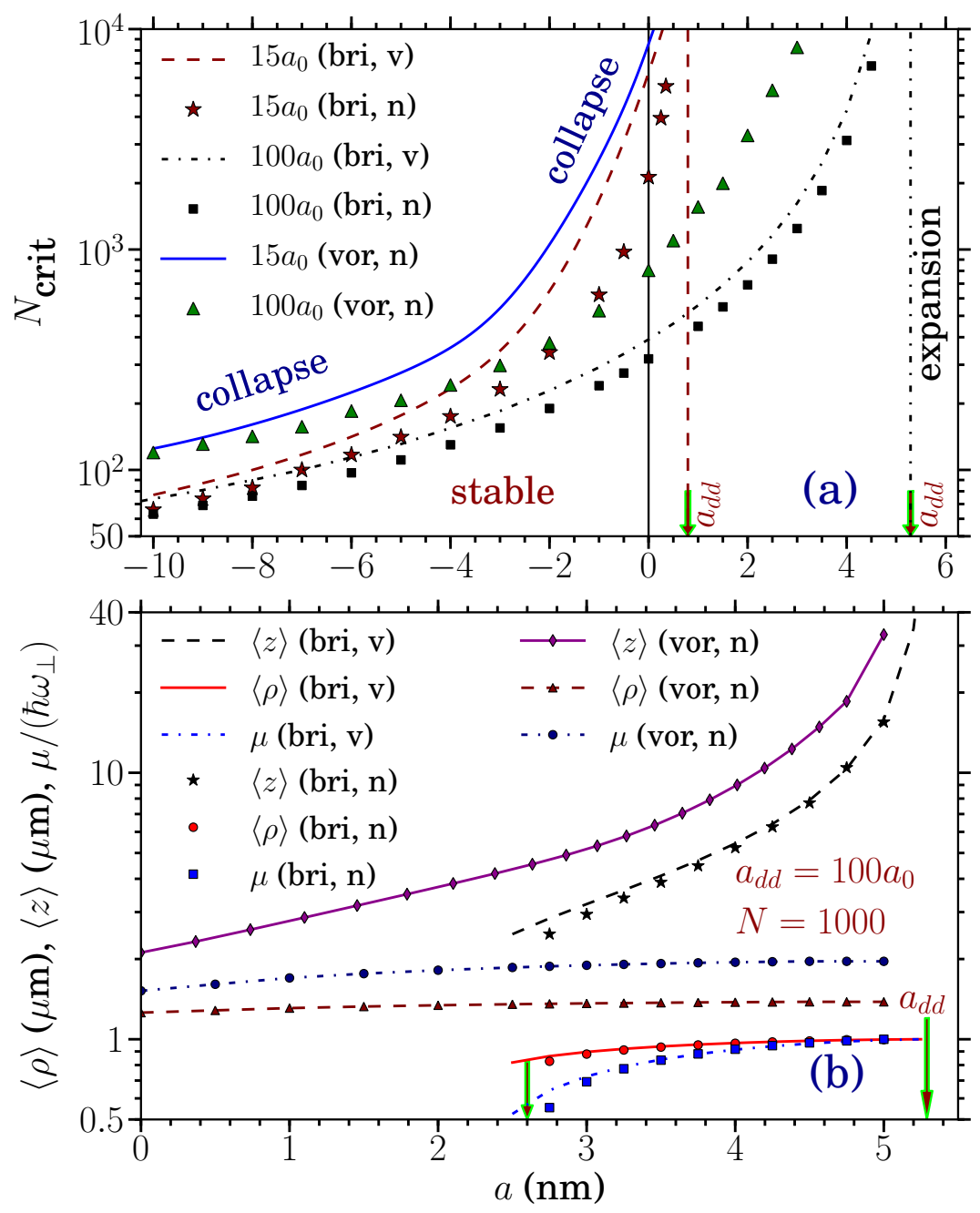

Figure 1. (a) $N_{\text {crit }}$ versus $a$ plot for $a_{d d}=15 a_{0}, 100 a_{0}$. (b) rms sizes $\langle\rho\rangle,\langle z\rangle$ and chemical potential $\mu$ versus $a$ for $N=1000$ and $a_{d d}=100 a_{0}$. The two arrows denote the region of stability of bright solitons. (variational: $\mathrm{v}$, numerical: $\mathrm{n}$, bright: bri, vortex: vor.)

$$
\ddot{w}_{z}=\frac{1}{w_{z}^{3}}+\frac{1}{\sqrt{2 \pi}} \frac{2 N}{w_{\rho}^{2} w_{z}^{2}}\left[a-a_{d d} h(\kappa)\right],
$$

with $g(\kappa)=\left[2-7 \kappa^{2}-4 \kappa^{4}+9 \kappa^{4} d(\kappa)\right] /\left(1-\kappa^{2}\right)^{2}, h(\kappa)=\left[1+10 \kappa^{2}-2 \kappa^{4}-9 \kappa^{2} d(\kappa)\right]\left(1-\kappa^{2}\right)^{2}$. Equations (4) and (5) determine the dynamics of bright solitons. The stationary widths are obtained from these equations by setting $\ddot{w}_{\rho}=\ddot{w}_{z}=0$. The stationary chemical potential $(\mu)$ is given by $\mu=1 / 2 w_{\rho}^{2}+1 / 4 w_{z}^{2}+w_{\rho}^{2} / 2+2 \mathcal{E}_{\text {dip }}$.

We perform a $3 \mathrm{D}$ numerical simulation in Cartesian $x, y, z$ variables employing imaginary- and real-time propagation with Crank-Nicolson method using Fortran programs provided in Ref. [27]. The dipolar interaction is evaluated by the usual fast Fourier transformation [7].

For bright solitons the system must be attractive. In the absence of dipole moment $\left(a_{d d}=0\right)$, attraction corresponds to $a<0$. For $a_{d d}>0$, the dipolar interaction in (1) 

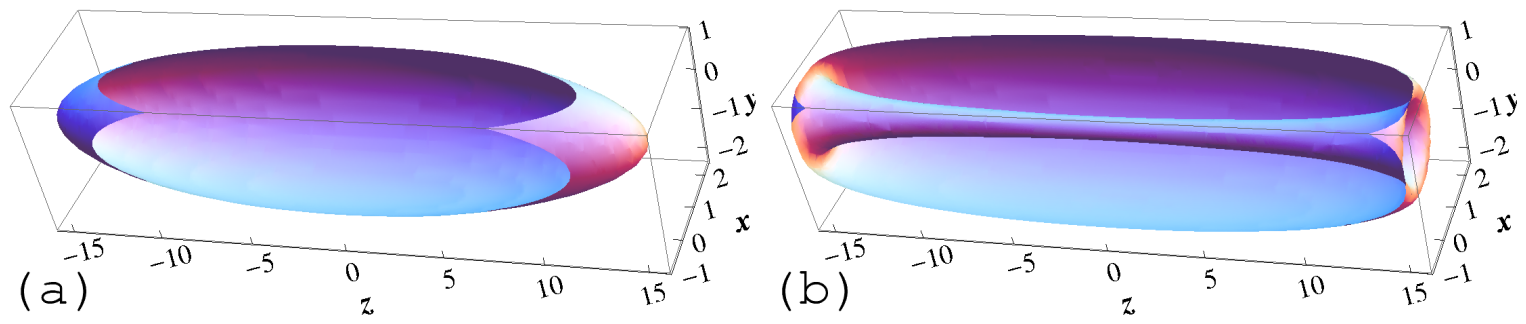

Figure 2. A sectional view of the $3 \mathrm{D}$ contour plot of density for a (a) bright $\left(a_{d d}=15 a_{0}\right.$ and $\left.a=0.5 \mathrm{~nm}\right)$ and (b) vortex $\left(a_{d d}=100 a_{0}, a=4 \mathrm{~nm}, l=1\right)$ solitons of $N=1000$ atoms. The density on the contour is 0.001 .

contributes attractively in the cigar shape, which can compensate for the atomic shortrange repulsion, thus forming bright solitons for $a>0$. From (5), the onset of attraction for a bright soliton is at $\left[a-a_{d d} h(\kappa)\right]=0$ while one must have $N \rightarrow \infty$. Then from (4), as $N \rightarrow \infty$, one must have $\left[2 a-a_{d d} g(\kappa)\right]=0$. These two conditions can be simultaneously satisfied as $\kappa \rightarrow 0$ for a weakly bound soliton of infinite extension along axial direction while $h(\kappa=0)=g(\kappa=0) / 2=1$. Consequently, bright solitons are allowed for $-\infty<a<a_{d d}$ and for $N$ below a critical number $N_{\text {crit }}$, as (4) and (5) only permit solution for $N<N_{\text {crit }}$. The system collapses for $N>N_{\text {crit }}$. These results are illustrated in figure 1 (a), where we show $N_{\text {crit }}$ versus $a$ for $a_{d d}=15 a_{0}\left({ }^{52} \mathrm{Cr}\right.$ atom), and $100 a_{0}$, for bright and vortex solitons from numerical and variational calculation. The value $a_{d d}=100 a_{0}$ is considered to simulate a possible DBEC of dipolar atoms (or molecules) of larger dipole moment, such as ${ }^{164} \mathrm{Dy}_{66}$, or ${ }^{166} \mathrm{Er}_{68}$, which are being considered for BEC experiment [28, 29]. Preliminary study [29] indicates that the dysprosium atom has a dipole moment of about nine times larger than that of ${ }^{52} \mathrm{Cr}$, which justifies the use of $a_{d d}=100 a_{0}$. The attractive region of collapse $\left(N>N_{\text {crit }}, a<a_{d d}\right)$, stable soliton formation $\left(N<N_{\text {crit }}, a<a_{d d}\right)$ and the repulsive region of expansion $\left(a>a_{d d}\right)$ are clearly shown in figure 1 (a). Due to the centrifugal repulsion, the vortex soliton can accommodate a larger number of atoms [24]. In our numerical simulation we shall consider DBEC with $a_{d d}>a>0$ - domain of soliton controlled solely by dipolar interaction and inaccessible for a normal BEC with $a_{d d}=0$. In figure 1 (b) we plot results for root-mean-square (rms) sizes $\langle\rho\rangle,\langle z\rangle$ and chemical potential $\mu$ of bright and vortex solitons. The variational results for bright solitons are in good agreement with the numerical results. From figures 1 (a) and (b) we find that the vortex soliton is stable in a wider domain of parameter space, due to the vortex core which keeps the atoms apart to avoid the collapse. Consequently, the vortex soliton is larger in size than the bright soliton with a larger chemical potential.

To illustrate the shape of the quasi-1D soliton, in figure 2 (a) we show a section of the 3D contour plot of density of a bright soliton of 1000 atoms for $a=0.5 \mathrm{~nm}$, and $a_{d d}=15 a_{0}=0.7938 \mathrm{~nm}$. A similar plot for the vortex soliton for $N=1000, a=4 \mathrm{~nm}$, and $a_{d d}=100 a_{0}=5.2917 \mathrm{~nm}$ is shown in figure 2 (b) which clearly exhibits the vortex core along the $z$ axis. 
Next we study by numerical (num) and variational (var) approaches the stability of the bright and vortex solitons shown in figure 2 under small breathing oscillation started by a sudden change in scattering length $a$. This can be implemented experimentally by the FR technique. The initial soliton was created by imaginary-time propagation and subsequent dynamics generated by real-time propagation. In figure 3 we plot rms sizes $\langle z\rangle$ and $\langle\rho\rangle$ versus time. For the breathing oscillation of $\langle\rho\rangle$, we also plot a model result calculated from the theoretical frequency of twice the trap frequency $\omega_{\perp}$ for an extreme cigar-shaped condensate using a hydrodynamic description [30]. For the bright soliton, the angular frequencies for $\langle\rho\rangle$ are $\omega_{\rho}=2 \omega_{\perp}$ (num,var, and theory). For the vortex soliton, $\omega_{\rho}=1.94 \omega_{\perp}$ (num) and $2 \omega_{\perp}$ (theory). The angular frequency for $\langle z\rangle$, controlled by the nonlinearity and not by any external trap, is very small in both cases.

Now we investigate the collision between two bright and two vortex solitons of figure 2. Two such solitons are placed at $z= \pm 25.6$ at $t=0$ and are then advanced by real-time propagation of (1) with $N=2000$. Each soliton is attributed a velocity of about $5 \mathrm{~mm} / \mathrm{s}$ towards center $z=0$ by including a phase factor $\exp (i v z)$ with $v$ a constant in the initial wave function. The real-time simulation is terminated when the solitons reach $z= \pm 25.6$ at $t=11.22 \mathrm{~ms}$. The collision dynamics is illustrated in figure 4 for bright and vortex solitons, where we show snapshots of contour plots of density $|\phi(x, 0, z, t)|^{2}$ at different times before (0, $\left.1.87 \mathrm{~ms}, 3.74 \mathrm{~ms}\right)$, during (5.61 ms) and after $(7.48 \mathrm{~ms}, 9.35 \mathrm{~ms}, 11.22 \mathrm{~ms})$ collision. The solitons come towards each other, interact at $z=0$ and then separate and come out practically unchanged. The quasielastic collision at such a large relative velocity of $1 \mathrm{~cm} / \mathrm{s}$ demonstrates the solitonic nature and robustness of the bright and vortex solitons. Video clips of the collision dynamics of figure 4 are also prepared for bright and vortex solitons and contained

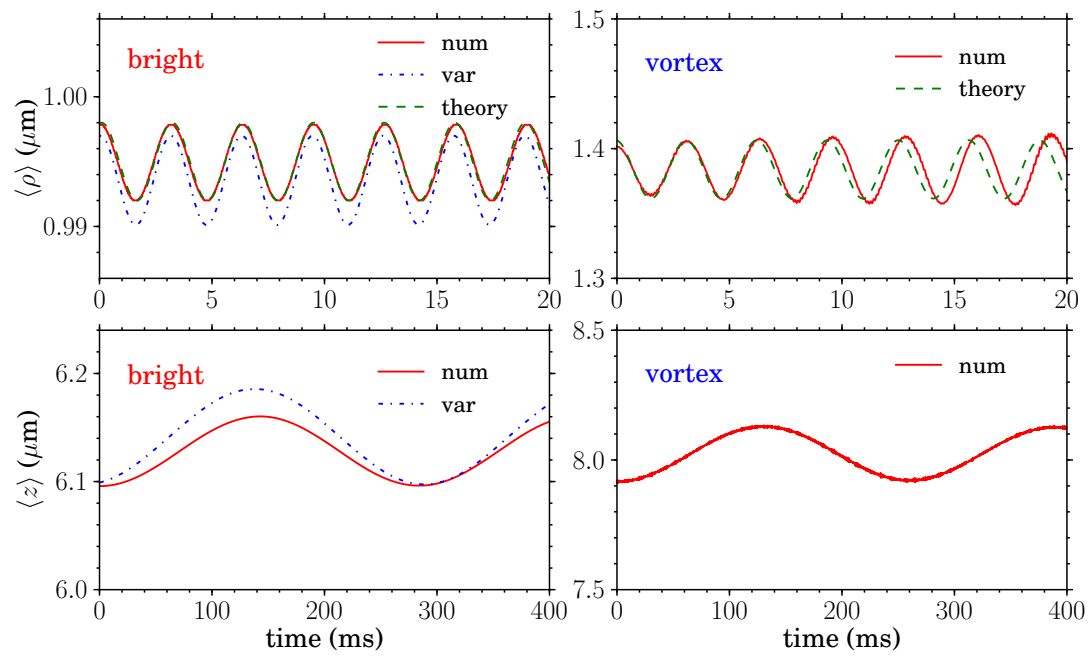

Figure 3. $\quad$ rms sizes $\langle z\rangle$ (lower panels) and $\langle\rho\rangle$ (upper panels) versus time during breathing oscillation of the bright (left panels) and vortex (right panels) solitons of figure 2 started by jumping $a$ from 0.5 to $0.5025 \mathrm{~nm}$ and from 4 to $4.02 \mathrm{~nm}$, respectively: numerical (num), variational [30] (var), theoretical (theory). 


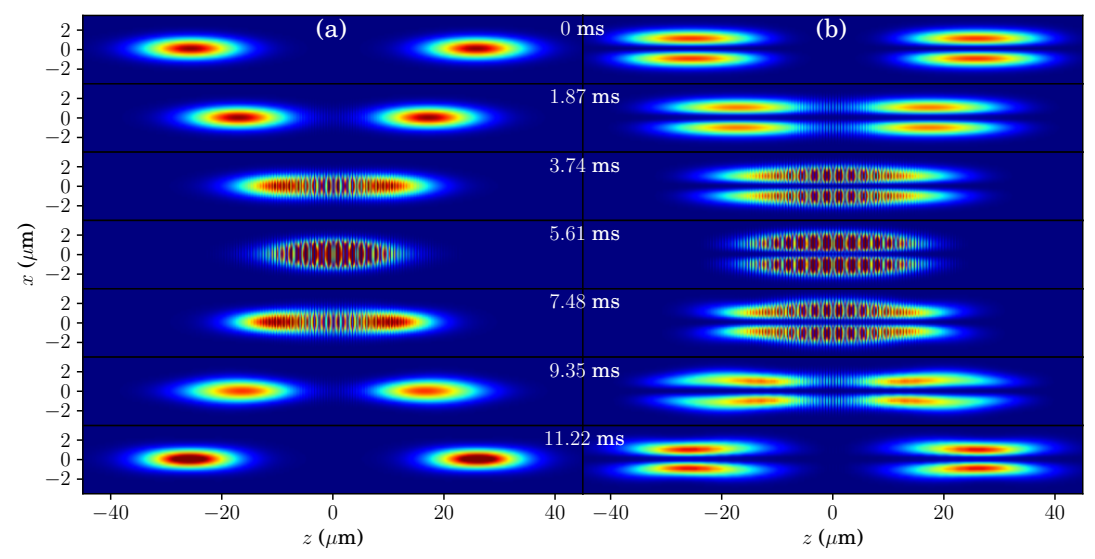

Figure 4. Contour plot of density $|\phi(x, 0, z, t)|^{2}$ of two colliding (a) bright and (b) vortex solitons of figure 2 with relative velocity $1 \mathrm{~cm} / \mathrm{s}$, before, during and after collision.
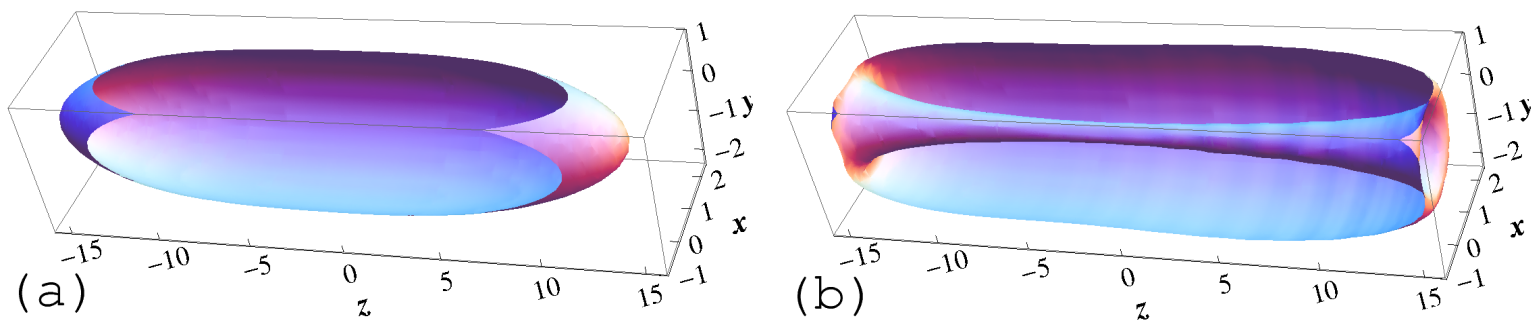

Figure 5. Same as figures 2 (a) and (b) at time $t=11.22 \mathrm{~ms}$ after the collision considered in figure 4

in supplementary clips S1 (also at http://www.youtube.com/watch?v=ds2CzZqN9M4) and S2 (also at http://www.youtube.com/watch?v=aQgLLhW08mE), respectively.

To illustrate the quasi-elastic nature of collision, we show in figures 5 (a) and (b) the $3 \mathrm{D}$ contour density plot of the final states at time $t=11.22 \mathrm{~ms}$ after the collision illustrated in figure 4 for bright and vortex solitons, respectively. The similarity of plots in figures 5 (a) and (b) for the final states after collision with the initial states in figures 2 (a) and (b) demonstrates the elastic nature of the collision. Specially, in figures 2 (b) and 5 (b) the radius of the vortex cores are practically the same determined by the fundamental healing length of the DBEC. To quantitatively compare the solitons before $(t=0)$ and after $(t=11.22 \mathrm{~ms})$ collision, we show in Table I the numerical and variational results of chemical potential, rms sizes, and number of atoms of the initial and final solitons, in good agreement with each other. There is no exchange of atoms between solitons during collision.

The quasi-elastic collision at high velocity is insensitive to the initial phase of the solitons. At low velocity, the interaction is sensitive to the initial phase. Two solitons placed side-by-side at rest along the $z$ direction of opposite phase repeal, whereas of the same phase attract and form a stable bound soliton molecule [31]. 
Table 1. Numerical (n) and variational (v) $\mu,\langle z\rangle,\langle\rho\rangle$, and $N$ of the solitons of figures 2 and 5 at $t=0$ and $11.22 \mathrm{~ms}$.

\begin{tabular}{|c|c|c|c|c|c|}
\hline & bright (v) & bright (n) & bright (n) & vortex $(\mathrm{n})$ & vortex $(\mathrm{n})$ \\
\hline$t$ & 0 & 0 & $11.22 \mathrm{~ms}$ & 0 & $11.22 \mathrm{~ms}$ \\
\hline$\mu$ & 0.9815 & 0.9822 & 0.9877 & 1.9686 & 1.9779 \\
\hline$\langle z\rangle$ & 6.0973 & 6.0958 & 6.0805 & 7.9749 & 8.0088 \\
\hline$\langle\rho\rangle$ & 0.9935 & 0.9924 & 0.9625 & 1.4018 & 1.4030 \\
\hline$N$ & 1000 & 1000 & 1000 & 1000 & 1000 \\
\hline
\end{tabular}

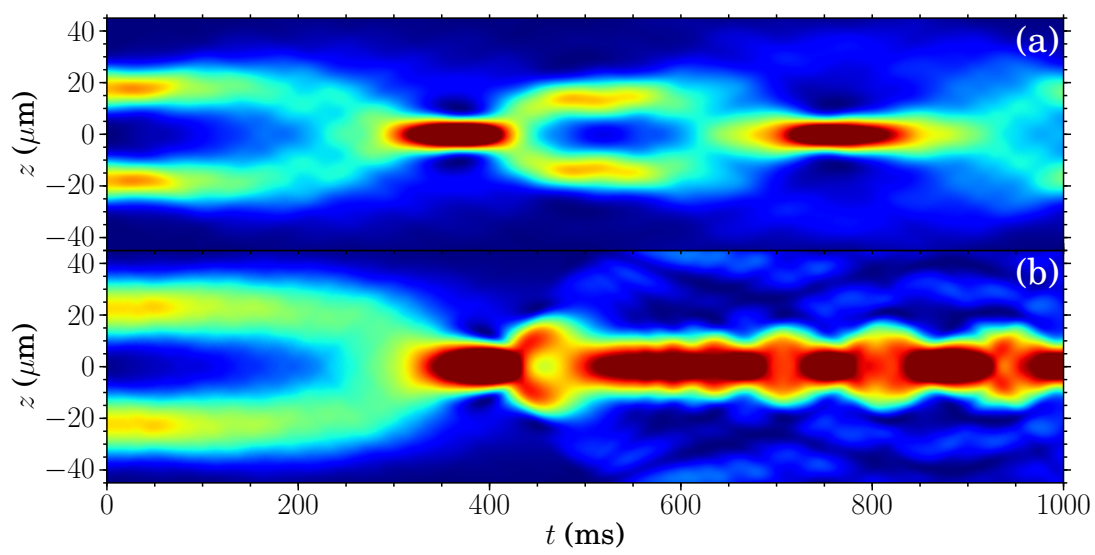

Figure 6. Dynamics of soliton pair formation from contour plot of 1D density versus time of (a) two bright and (b) vortex solitons of figure 2 placed side by side at rest.

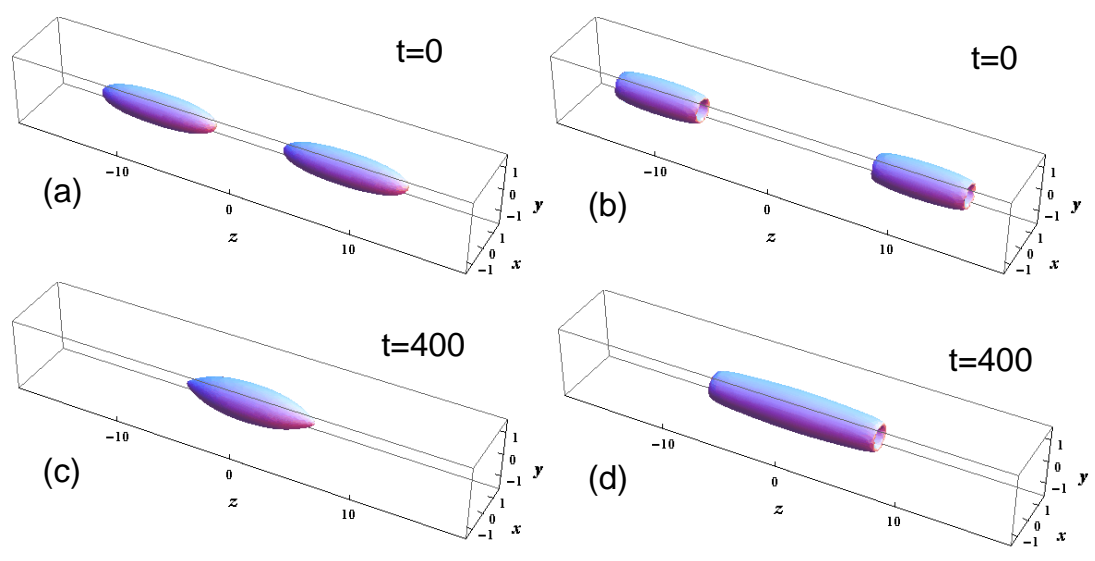

Figure 7. The initial 3D contour plots of the two (a) bright and (b) vortex solitons of Fig. 6 at $t=0$ and of the two soliton molecules formed of the two (c) bright and (d) vortex solitons at $t=400 \mathrm{~ms}$. The vortex cores can clearly be seen in (b) and (d). 
The dynamics of the formation of a soliton molecule from two equal-phase solitons of figure 2 is shown in figure 6, where we exhibit the contour plot of $1 \mathrm{D}$ density $d(z, t) \equiv 2 \pi \int \rho d \rho|\phi(\mathbf{r}, t)|^{2}$ versus time $t$ and $z$. The initial positions of the bright (vortex) solitons are $\pm 17.6 \mu \mathrm{m}( \pm 22.4 \mu \mathrm{m})$. Due to dipolar attraction the solitons come close, coalesce and oscillate forming a stable bound soliton molecule [31. In the case of the two bright solitons in Fig. 6 (a), after formation, the soliton molecule breaks into two solitons, then forms a molecule, thus oscillating between a molecule and twosoliton phases. In case of the collision of two vortex solitons, the final soliton molecule is a vortex molecule with zero density along the axial direction. This is illustrated in Fig. 7 where we show the 3D contour plots of the two interacting bright and vortex solitons of Fig. 6 at $t=0$ and $400 \mathrm{~ms}$. A pair of bright solitons, bound together by a dark soliton, has been observed in dispersion-managed fiber optics [32]. Now it seems possible to observe the present soliton molecules in DBEC. It should be possible to consider an antivortex [33] in a DBEC using the present model based on a 3D GP equation. A problem of future interest is to consider the collision between a vortex and antivortex at low velocities. One may expect that a vortex-antivortex pair will annihilate the angular momentum quantum number, thus forming after collision a soliton without angular momentum, instead of forming vortex molecule of the type shown in Fig. 7 (d) with a hollow along the axial direction. Video clips of the molecule formation dynamics of figure 6 are also prepared for bright and vortex solitons and contained in supplementary clips S3 (also at http://www.youtube.com/watch?v=tnnF0nbilgs) and S4 (also at http://www.youtube.com/watch?v=01AWIBzbrDs), respectively.

To conclude, we suggested the possibility of bright and vortex solitons in DBEC for repulsive short-range interaction for $a<a_{d d}$ and studied the statics (rms sizes and chemical potential) and dynamics (collision and breathing oscillation) of such solitons. Numerical results of stability phase plot and breathing oscillation of the bright solitons are in good agreement with variational calculations. The collision between two solitons is quasi-elastic at high velocities $(\sim 1 \mathrm{~cm} / \mathrm{s})$ and independent of initial phase. At very low $(\sim 0)$ initial velocity, two equal-phase solitons placed side-by-side at rest form a bound soliton molecule [31] due to the dipolar attraction. With present technology, these solitons could be created in laboratory and the present theoretical results verified.

\section{Acknowledgments}

We thank FAPESP (Brazil), CNPq (Brazil), DST (India), and CSIR (India) for partial support.

\section{References}

[1] Strecker K E, Partridge G B, Truscott A G and Hulet R G 2002 Nature 417150

[2] Khaykovich L, Schreck F, Ferrari G, Bourdel T, Cubizolles J, Carr L D, Castin Y and Salomon C 2002 Science 2561290

[3] Cornish S L, Thompson S T and Wieman C E 2006 Phys. Rev. Lett. 96170401 
[4] Inouye S et al. 1998 Nature 392, 151

[5] Koch T, Lahaye T, Metz J, Frohlich B, Griesmaier A and Pfau T 2008 Nature Phys. 4218 Lahaye T et al. 2007 Nature 448672

Lahaye T et al. 2008 Phys. Rev. Lett. 101080401

Stuhler J, Griesmaier A, Koch T, Fattori M, Pfau T, Giovanazzi S, Pedri P and Santos L 2005 Phys. Rev. Lett. 95150406

Griesmaier A, Stuhler J, Koch T, Fattori M, Pfau T and Giovanazzi S 2006 Phys. Rev. Lett. 97 250402

[6] Lahaye T, Menotti C, Santos L, Lewenstein M , and Pfau T 2009 Rep. Prog. Phys. 72126401

[7] Góral K and Santos L 2002 Phys. Rev. A 66023613

[8] Ronen S, Bortolotti D C E and Bohn J L 2007 Phys. Rev. Lett. 98030406

Wilson R M, Ronen S, Bohn J L and Pu H 2008 Phys. Rev. Lett. 100245302

Saito H, Kawaguchi Y and Ueda M 2009 Phys. Rev. Lett. 102230403

[9] Dutta O and Meystre P 2007 Phys. Rev. A 75053604

Parker N G, Ticknor C, Martin A M and O'Dell D H J 2009 Phys. Rev. A 79013617

Wilson R M, Ronen S and Bohn J L 2009 Phys. Rev. A 80023614

[10] Santos L, Shylapnikov G V, Zoller P and Lewenstein M 2000 Phys. Rev. Lett. 851791

[11] Gligorić G, Maluckov A, Hadzievski L and Malomed B A 2009 J. Phys. B: At. Mol. Opt. Phys. 42145302

[12] Giovanazzi S, Gorlitz A and Pfau T 2002 Phys. Rev. Lett. 89130401

O'Dell D H J, Giovanazzi S and Eberlein C 2004 Phys. Rev. Lett. 92250401

[13] Yi S and You L 2001 Phys. Rev. A 63053607

[14] Salasnich L, Malomed B and Toigo F 2007 Phys. Rev. A 76063614

[15] Kawaguchi Y and Ohmi T 2004 Phys. Rev. A 70043610

[16] Adhikari S K 2003 New J. Phys. 5137 Adhikari S K 2004 Few-Body Systems 34197

[17] Pedri P and Santos L 2005 Phys. Rev. Lett. 95200404 Nath R, Pedri P and Santos L 2009 Phys. Rev. Lett. 102050401

[18] Tikhonenkov I, Malomed B A and Vardi A 2008 Phys. Rev. A 78043614

[19] Tikhonenkov I, Malomed B A and Vardi A 2008 Phys. Rev. Lett. 100090406

[20] Baizakov B B, Abdullaev F K, Malomed B A and Salerno M 2009 J. Phys. B: At. Mol. Opt. Phys. 42175302

[21] Cuevas J, Malomed B A, Kevrekidis P G and Frantzeskakis D J 2009 Phys. Rev. A 79053608

[22] Sinha S and Santos L 2007 Phys. Rev. Lett. 99140406

[23] Perez-Garcia V M, Michinel H and Herrero H 1998 Phys. Rev. A 573837

[24] Adhikari S K 2001 Phys. Rev. E 65016703

[25] Dalfovo F and Stringari S 1996 Phys. Rev. A 532477

[26] Yi S and You L 2004 Phys. Rev. Lett. 92193201

[27] Muruganandam P and Adhikari S K 2009 Comput. Phys. Commun. 1801888

[28] Hancox C I, Doret S C, Hummon M T, Luo L J and Doyle J M 2004 Nature 431281 McClelland J J and Hanssen J L 2006 Phys. Rev. Lett. 96143005

[29] Youn S H, Lu M W, Ray U and Lev B L 2010 Phys. Rev. A 82043425

[30] Stringari S 1996 Phys. Rev. Lett. 772360

[31] Al Khawaja U, Stoof H T C, Hulet R G, Strecker K E and Partridge G B 2002 Phys. Rev. Lett. 89200404

Al Khawaja U 2010 Phys. Rev. E 81056603

[32] Stratmann M et al. 2005 Phys. Rev. Lett. 95143902

[33] Liu M, Wen L H, Xiong H W and Zhan M S 2006 Phys. Rev. A 73063620 